\title{
A Quadrature-Based A pproach to Improving the Collocation Method for Splines of Even Degree' ${ }^{1}$ )
}

\author{
IAN.H. SloAN and W. L. WENDland \\ Dedicated to Prof. Dr. S. G. Mikhlin on the occasion of his 80th birthday
}

Die ,Qualokationsmethode“ ist eine kürzlich entstandene durch numerische Quadratur modifi. zierte Kollokationsmethode. Sie wird hier auf eine Klasse, von Randintegralgleichungen angewendet, wobei Spline-Funktionen stückweiser Polynome von geradem Grad als Ansatzfunktionen benutzt werden. Die analysierten Probleme sind von der Form $(L+K) u=f$, wobei $L$ ein Faltungsoperator mit gorndem Symbol und $K$ ein'Operator mit stärkeren Glättungseigenschaften als $L$ ist. Wir zeigen, wie für einen Ansatzraum der Dimension $n$ spezielle $2 n$-Punkt-Quadraturformeln konstruiert.werden können, welche die komponierten Zweipunktformeln von Gau $B$ verallgemeinern, so daB eine stabile konvergente Qualokationsmethode entsteht, deren Konvergenzordnung in geeigneten Sobolev-Räumen negativer Ord́nung höher ist als die der gewöhnlichen Mittelpunktkollọkation, die kürzlich von Saranen analysiert. wurde. Mit Hilfe der Technik glatter Störungen wird darüber hinaus hier auch die Analysis der Qualokationsmethoden mit Splines ungeraden Grades, die von Sloan entwickelt wurde, verallgemeinert.

Развитый недавно метод квалокации является методом коллокации, модифицирова̀нным квадратурными формулами. Этот метод здесь применяется к одному классу граничных интегральных уравнении, причём в качестве базисных фунікций используют полиномиальные сплайн-фуннций чётной степени. Рассматриваемые задачи пмеют вид $(L+K) u=f$ где $L$ оператор в свёртках с чётным симболом, а $K$ оператор, обладающий более сильным свойством сглаживания чем $L$. Мы покажем, как можно построить для базисного пространства размерности $n 2 n$-точечные квадратурные формулы, обобщающие составные двух-точечные формулы Гаусса, чтобы получить устойчивый и сходящип̆ся метод квалокации. Порядок сходимости этого метода в подходящих пространствах Соболева отрицательного порядка выше чем в случае обычной коллокации-в средней точке, изучаемон̆ недавно Saranen. Кроме того обобщается с помощью техники гладких возмущений анализ методов кралокации со сплайн-функциями шечётной степени, развитый Sloan.

The "qualocation:" method, a recently proposed quadrature-based extension of the collocation method, is here applied to a class of boundary integral equations, using an even degree spline trial space on a uniform partition. The problems handled are of the form $(L+K) u=f$, where $L$ is a convolutional operator with even symbol, and $K$ is an operator with a greater smoothing effect than $L$. For a trial space of dimension $n$, it is shown that a certain $2 n$-point quadrature rulé, which is a generalization of the repeated 2-point Gauss rule, gives ${ }^{-}$a stable qualocation method, and yields an order of convergence, in suitable negative norms, two powers of $h$ higher than achieved by the mid-point collocation method in the recent analysis of Suranen. The treatment of the smooth, perturbation covers also the earlier analysis of the odd degree spline case by Sloan.

1) Acknowledgement. This work was carried out while the first author was supported as Gastprofessor at the University of Stuttgart by the Deutsche Forschungsgemeinschaft, under the contract number Stu $23 / 70-1$. The support of the DFG is gratefully acknowledged. 


\section{Introduction}

In a previous paper [14] a general class of methods ('qualocation methods') was proposed for solving a special class of boundary integral equations.

$$
L u=f \text {. }
$$

There a specific method was developed for a particular class of strongly elliptic boundary integral equations with even principal symbol, for trial spaces of odd degree smoothest splines on: a uniform mesh. Here we consider, for a slightly more general class of problems, the case of even degree smoothest splines; for example piecewise-constant functions, or piecewise-quadratics with continuous first, deripative.

In brief, a qualocation method requires the choice of a 'trial' space $\dot{S}_{h}$, here a spline space; a 'test' space $T_{h}$ ' of the same finite dimension $n_{h}$; and a quadrature rule $Q_{h}$ of the form $Q_{h} g=\sum_{l=1}^{m_{n}} w_{l} g\left(t_{l}\right)$, with $m_{h} \geqq n_{h}$. The method is: find $u_{h} \in S_{h}$ such that

$$
Q_{h}\left(\bar{\chi}_{h} L u_{h}\right)=Q_{h}\left(\bar{\chi}_{h} f\right) \quad \forall \dot{\chi_{h}} \in T_{h}
$$

It is shown in [14] that if $m_{h}=n_{h}$ the method is equivalent to the collocation method with trial space $S_{h}$ and collocation points $t_{1}, \ldots, t_{n_{n}}$. Thus in general the method may be thought of as a quadrature-based generalization of the collocation method; hence the name 'qualocation'. It may also be thought of as a semi-discrete version of the Petrov-Galerkin method, or'a generalization of quadrature formula methods [9]. The essential point is that the freedom that exists in the design of the quadrature rule may be exploited to improve the order of convergence, especially in 'negative norms', over that achievable with the collocation method. Hence, qualocation can provide higher order convergence than collocation. As we shall see, the order of convergence can even exceed that of the superconvergent Galerkin or corresponding. Galerkincollocation methods $[3,6]$. Of course one also has to establish the stability of the resultant method, and this is a central issue in the analysis.

In the present work, as in [14], the functions $u$ and $f$ in (1.1) are taken to be1periodic, and $L$ is a pseudodifferential operator of real order $\beta$. Here we first consider operators with even symbol, given in Fourier series form [12] by

$$
L u(x)=\hat{u}(0)+\sum_{k \neq 0}|k|^{\beta} \hat{u}(k) \mathrm{e}^{2 \dot{x} 1 k x}, \quad \text { where } \hat{u}(k)=\int_{0}^{1} u(x) \mathrm{e}^{-2 \dot{\pi} 1 k x} d x
$$

and then in Section 6 consider smooth perturbations $(L+K) u$ of (1.3). As discussed in [14], the logarithmic potential on a circle is obtained by setting $\beta=-1$ in (1.3); and the choice $\beta=0$ makes $L$ the identity, in which case the collocation method reduces to spline interpolation.

As indicated above, in this paper we choose the trial space $S_{h}$ to be $S_{h}{ }^{d} \subset C^{d^{\prime}-1}$, the space of smoothest splines of even degree $d$, subordinate to the uniform mesh spacing $h=1 / n_{h}=1 / n$. The breakpoints are chosen to be

$$
\left\{j h: j=0,1, \ldots, n^{\prime}-1\right\} \text {. }
$$

For $d=0$ the space $S_{h}$ consists of piecewise-constant functions.

As in [14], the test space $T_{h}$ is taken to be the trigonometric space

$$
T_{h}=\operatorname{span}\left\{\mathrm{e}^{2 \pi i p x}:-n / 2<p \leqq \bar{n} / 2, p \in \mathbf{Z}\right\} \text {. }
$$


(In practice a slight modification of $T_{A}$, as in Section 5 of [14], is useful, to guarantee that $u_{h}$ is real). This choice of test space gives high orders of convergence for the Petrov-Galerkin method, according to the analysis of [2]. There, however, quadrature was not considered.

The most interesting question concerns the choice of the quadrature rule $Q_{h}$. If we choose a rule with $n$ points (i.e. one point per sub-interval) then, as noted already, the method is equivalent to a collocation method. Hence the simplest non-trivial qualocation method that respects the rotational symmetry of the test and trial spaces uses two points per sub-interval; and may be expressed as

$$
Q_{n} g=\frac{1}{n} \sum_{j=0}^{n-1}\left[w_{1} g \cdot\left(\frac{j+\varepsilon_{1}}{n}\right)+w_{2} \dot{g}\left(\frac{j+\varepsilon_{2}}{n}\right)\right],
$$

where $0 \leqq \varepsilon_{1}<\dot{\varepsilon}_{2} \leqslant 1$, and $w_{1}+w_{2}=1$.

The question, then, is how to choose the free parameters $\varepsilon_{1}, \varepsilon_{2}$ and $w_{2}$ in (1.6), in order to obtain high orders of convergence. In [14], for the case of splines of odd degree, we made the simple choice $\varepsilon_{1}=0, \varepsilon_{2}=1 / 2$, leaving only the weight $w_{2}$ to be fixed. However, for the splines of even degree it seems less obvious that the breakpoints are desirable quadrature points. Consequently, in the present work we avoid a priori assimptions about $\varepsilon_{1}$ and $\varepsilon_{2}$.

The main results of the paper are contained in Theorem 2.1 in the next section. There are three parts of the theorem, the successive parts being concerned with increasing levels of specialization in the choice of the quadrature parameters.

The first part of the theorem states a comforting result; that almost every sensible choice of the quadrature parameters yields a stable method that has the same optimalorder rate of convergence as predicted by SARANEN and WENDLAND [11] and ARNOLD and WENDLAND [4] for the mid-point collocation method and a more general class of operators $L$. The only restrictions are that one of the quadrature-point parameters, say $\varepsilon_{2}$; lies in the interior of the interval $[0,1]$ and has an associated positive weight, and that the other weight is non-negative. For the general choice of quadrature parameters the highest rate of convergence predicted by the first part of Theorem 2.1 , as in [4] for the collocation method, is

$$
\left\|u_{h}-u\right\|_{\beta} \leqq C h^{d+1-\beta}\|u\|_{d+1}^{\prime} .
$$

Here $\|u\|_{s}$ denotes the norm in the periodic Soboler space $H^{s}$, which cán be defined bý

$$
\|u\|_{s}^{2}=|\hat{u}(0)|^{2}+\sum_{k \neq 0}|k|^{2 s}|\dot{u}(k)|^{2}
$$

and $C$, here às elsewhere in the paper; denotes a generic constant, which may take different values at its different occurrences. A's a special case of Theorem 2.1(i), we recover (by setting $w_{1}=0$, and $\varepsilon_{2}=\varepsilon$ ) a known result (see Theorem 3 of ScHMmT, [13]), namely that the $\varepsilon$-collocation method of [13], which is the collocation method with the collocation points $\{(j+\varepsilon) h: j=0,1, \ldots, n-1\}$, is stable for the operator (1.3), and yields the order of convergence (1.7), for all values of $\varepsilon$ in the open interval $(0,1)$.

The second part of Theorem 2.1 asserts that a quadrature rule that is 'symmetric about the mid-point' can yield a higher order of convergence, in an appropriate negative norm. Precisely, the error bound-with the highest possible power of $h$ now becomes -

$$
\begin{aligned}
& \left\|u_{h}-u\right\|_{\beta_{-1}} \leqq C h^{d+2-\beta}\|u\|_{d+2} . \\
& \text { extra power of } h \text { is achieved. }
\end{aligned}
$$

Thus one extra power of $h$ is achieved. However, this is at the expense of a more restrictive smoothness requirement on $u$ on the right of (1.9), and a 'more-negative' 
norm on the left. For the special case of mid-point collocation (i.e. $\varepsilon_{1}=\varepsilon_{2}=1 / 2$ ) the result (1.9) has already been obtained by SARANEN in [10]. Thus the second part of Theorem 2.1 may be thought of as a generalization of Saranen's result to more general symmetric qualocation methods.

The most interesting part of Theorem 2.1 (most interesting because it offers the possibility of an improved approximation) is the last: for this asserts that one particular choice of symmetric quadrature rule can lead to yet two more powers of $h$ in appropriate cases. In addition to the bounds (1.7) and (1.9), for this special choice we have also

$$
\left\|u_{h}-u\right\|_{\beta-3} \leqq C h^{d+4-\beta}\|u\|_{d+4} \cdot
$$

For example, for the piecewise-constant trial space (i.e. $d=0$ ) and the logarithmic o potential (i.e. $\beta=-1$ ) we obtain $O\left(h^{5}\right)$ convergence, provided $u . \epsilon \cdot H^{4}$, and provided we are willing to look at errors in the $H^{-4}$ norm. A striking reflection is that the Galerkin method for the same problem yields at best $O\left(h^{3}\right)$ convergence - see, for example, [7]. On the other hand it should be said that the Galerkin result imposes a weaker 'smoothness requirement on $u$ (namely $u \in H^{1}$ ), and is achieved in a much less-negative norm (namely $\mathrm{H}^{-2}$ ).

Theorem 2.1 is proved in Sections 3 and 4.

The special quadrature parameters in the third part of the theorem, yielding the higher rate of convergence shown in (1.10), are defined as follows: $w_{1}=w_{2}=1 / 2$, $\varepsilon_{1}=x_{0}$, and $\varepsilon_{2}=1-x_{0}$, where $x_{0}$ is the unique zero in the interval $(0,1 / 2)$ of the function

$$
G_{y}(x)=\sum_{n=1}^{\infty} n^{-y} \cos 2 \pi n x,
$$

with $\bar{\gamma}=d+2-\beta$. The properties of this function are discussed in Section 5 . Here we record some values of $x_{0}(\gamma)$ so that they are available for application.

\begin{tabular}{|c|c|c|}
\hline & $\gamma$ & $x_{0}(\gamma)$ \\
\hline- & 2 & $1 / 2-1 / 2 / \sqrt{3}=0.2113248654$ \\
\hline- & 3. & 0.2308296503 \\
\hline & 4 & $1 / 2-(1 / 4-1 / \sqrt{30})^{1 / 2}=0.2403351888$ \\
\hline & 5 & 0.2451188417 \\
\hline & $\infty$ & 0.25 \\
\hline
\end{tabular}

Table 1. Least positive zeros of the function $G_{y}$ defined by (1.11)

The following remarks may help to give some insight into the quadrature parameters in Table 1. First, for $\gamma=2$ the points $x_{0}$ and $1-x_{0}$ are the abscissas of the 2 -point Gauss rule, shifted to the interval $[0,1]$. The value $\gamma=2$ is the appropriate value of $\gamma$ if we have $\beta=0$, in which case $L$ is the identity operator, and $d=0$. Now in this case the integrals to be approximated, if we adopt the. Petrov-Galerkin view, are discontinuous piecewise-smooth functions, and for such functions the two-point Gauss rule would seem to be an ideal choice. Second, if $d$ is very large, or if the order $\beta$ has a large negative value, then the function $L u_{h}$ becomes a very 
smooth periodic function, for which the equal weight and nearly equally-spaced rule that is achieved when $\gamma$ is large would seem entirely appropriate. The other values in Table 1 may be thought of as intermediate between these two extremes.

The treatment of the perturbations $L+K$ in Section 6 is expressed in sufficiently general terms to cover not only the present method but also that in [14], and other qualocation methods. An important consequence of the perturbation result in Section 6 is that the conclusions expressed in the following theorem, and in the corresponding result in [14], hold not only for uniform meshes on circles, but also for smoothly varying meshes on' smooth closed curves (see ['4]). Hence, the qualocation methods could be applied to all the examples given in [6], and would converge with the high orders predicted here and in [14].

Acknowledgements: The authors express their gratitude to G. C. Chandler for providing us with the nontrivial entries of Table 1 , and to G. Brown and D. Wilson of the University of New South. Wales for proving for us, in [5], that $G_{y}$ in (1.11) has a unique zero on $(0,1 / 2)$.

\section{The main result}

Theorem 2.1: Let $\beta$ be a real number, and let $L$ be as in (1.3). Let $d>\beta-1 / 2$ be $a$, non-negative even integer, and let $S_{h}=S_{h}{ }^{d}$ be the space of smoothest splines of degree $d$. with breakpoints (1.4). Let $T_{h}$ be the trigonometric function space given by (1.5), and let. : $Q_{h}$ be the quadrature rule (1.6), where $0<w_{2} \leqq 1, w_{1}=1-w_{2}, 0<\varepsilon_{2}<1$ and $0 \leqq \varepsilon_{1}<1$. Then the qualocation equation (1.2), with $/$ any continuous function, is uniquely solvable for $u_{h} \in S_{h}$.

Assume in addition that $s$ and $t$ are real numbers satisfying $s \leqq i, s<d+1 / 2$, $\beta+1 / 2<t$; and that the solution $u$ of (1.1) belongs to $H^{t}$.

(i) For $\beta \leqq s$ and $t \leqq d+1$, there holds the estimate

$$
\left\|u_{h}-u\right\|_{s} \leqq C h^{t-s}\|u\|_{l} \text {. }
$$

(ii) If the quadrature rule is symmetric, in the sense that $w_{1}=w_{2}=1 / 2$ and $\varepsilon_{2}=$ $1-\varepsilon_{1}$, then there holds the estimate

$$
\left\|u_{h}-u\right\|_{s} \leqq C h^{\min (t-8, t-\beta, d+1-s, d+2-\beta)}\|u\|_{t} \text {. }
$$

(iii) If $w_{1^{\prime}}=w_{2}=1 / 2$ and $\varepsilon_{2}=1-\varepsilon_{1}$, and if $\varepsilon_{1}$ is, the least positive zero of the function $G_{d+2-\beta}^{\prime}$, where

$$
G_{y}(x)=\sum_{n=1}^{\infty} n^{-\gamma} \cos 2 \pi n x,
$$

then there holds the estimate

$$
\left\|u_{h}-u\right\|_{s} \leqq C h^{\min (t-s, t-\beta, d+1-s, t+4-\beta)}\|u\|_{t} .
$$

Remark 1: Without loss we may restrict $s$ and $t$ in (2.2) by $\beta-1 \leqq s \leqq t \leqq d+2$ and in (2.4) by $\beta-3 \leqq s \leqq i \leqq d+4$.

Remark 2: Since the condition $d>\beta-1 / 2$, which is modelled on [4], is not strong enough to guarantec absolute convergence of the Fourier series for $L u_{h}$ (see Section 3), care is needed in defining the sense of convergence. We shall always understand the convergence to be in the conventional sense for the pointwise convergence of Fourier series, namely.

$$
\sum_{k}=\lim _{L \rightarrow \infty} \sum_{|k|<L} \text {. }
$$




\section{Proof of Theorem 2.1 - first stage}

Following [4] and [14], it is convenient to define $\Lambda_{n}=\{p \in \mathrm{Z}:-n / 2<p \leqq n / 2\}$. Then the test space $T_{h}^{\prime}$ becomes $T_{h}=\operatorname{span}\left\{\varphi_{p}: p \in A_{n}\right\}$, with $\varphi_{p}(x)=\mathrm{e}^{2 \pi k p x}, p \in \mathbf{Z}$, and the method becomes: find $u_{h} \in S_{h}{ }^{d}$ such that

$$
Q_{h}\left(\bar{\varphi}_{p} L u_{h}\right)=Q_{h}\left(\bar{\varphi}_{p} L u\right), \quad p \in A_{n},
$$

in the right-hand side of which we have replaced $f$ by $L u$.

As in [14], the sum (1.3) for $L u(x)$ converges absolutely because $\beta+1 / 2<t$, thus the right-hand side of (1.3) is well defined, and we have

$$
Q_{h}\left(\bar{\varphi}_{p} L u\right)=\hat{u}(0) Q_{h}\left(\bar{\varphi}_{p}^{-}\right)+\sum_{k \in \mathbf{Z}_{j}^{*}}|k|^{\beta} \hat{u}(k) Q_{h}\left(\bar{\varphi}_{p} \varphi_{k}\right)
$$

where $Z^{*}=Z \backslash\{0\}$. By direct application of (1.6), or by symmetry, we find

$$
Q_{h}\left(\bar{\varphi}_{p} \varphi_{k}\right) \doteq 0 \quad \text { if } \quad k \neq^{\prime} p(\bmod n)
$$

For the non-vanishing quadrature sums we define.

$$
e_{l}=Q_{h}\left(\bar{\varphi}_{p} \dot{\varphi}_{p+i n}\right)=Q_{h}\left(\dot{\varphi}_{l n}\right)
$$

and find, using the quadrature rule (1.6),

$$
\ddot{e}_{l}=w_{1} \mathrm{e}^{2 \pi 1 \varepsilon_{1}}+w_{2} \mathrm{e}^{2 \pi 1 / \varepsilon_{1}} \text {. }
$$

Then the right-hand side of (3.1) becomes

$$
Q_{n}\left(\bar{\varphi}_{p} L u\right)= \begin{cases}\dot{u}(0)+\sum_{l \in \mathbf{Z}^{*}}|\ln |^{\beta} \hat{u}(\ln ) e_{l}, & p \doteqdot 0 \\ |p|^{\beta} \hat{u}(p)+\sum_{l \in \mathbf{Z}^{*}}|p+\ln |^{\beta} \hat{u}(p+l n) e_{l}, & \dot{p} \in \dot{\Lambda}_{n}^{*}\end{cases}
$$

where $\dot{A}_{n}^{*}=\Lambda_{n} \backslash\{0\}$.

Now consider the left-hand side of (3.1). The analogue of (1.3) is

$$
\dot{L} u_{h}(x)=\hat{u}_{h}(0)+\sum_{k \in \mathbf{Z}^{*}}|k|^{\beta} \hat{u}_{h}(k) \mathrm{e}^{2 \pi \mathrm{i} k x} \text {. }
$$

Because $u_{h}$ is a spline of even degree $d$, its Fourier coefficients satisfy the following recurrence relation (see $[4$, equation $(2.7)]$, with appropriate phase adjustment to cater for the different choice of breakpoints):

Thus

$$
(p+\ln )^{d+1} \hat{u}_{h}(p+l n)=p^{\dot{d}+1} \hat{u}_{h}(p), p, l \in \mathbf{Z} \text {. }
$$

$$
\hat{u}_{h}(p+l n)= \begin{cases}0, & p=0, l \in \mathbf{Z}^{*} \\ \frac{p^{d+1}}{(p+l n)^{d+1}} \hat{u}_{h}(p), & p \in \Lambda_{n}^{*}, l \in \mathbf{Z}^{\prime}\end{cases}
$$

and we may write (3.6) as

$$
\begin{aligned}
& L u_{h}(x)=\hat{u}_{h}(0)+\sum_{p \in A_{n}} \hat{u}_{h}(p)\left[|p|^{\beta} \mathrm{e}^{2 \pi i p x}\right. \\
& \left.+p^{d+1} \sum_{l \in \mathbf{Z}^{*}} \operatorname{sign} l \dddot{\mid} p+\left.\ln \right|^{\beta-d-1} \mathrm{e}^{2 \pi 1(p+\ln ) x}\right] \text {. }
\end{aligned}
$$

Because we assume only $d>\beta-1 / 2$, the sum over $l$ is not absolutely convergent, and therefore care is needed in forming the partial sums. The convergence is always understood in the sense of (2.5); that is, the symmetric partial sums are used. (Note that (3.6) and (3.8) are equivalent when interpreted in this sense, because the individual terms in the sum over $l$ in the latter converge to zero.) 
We now show that the series (3.8) for $\ln (x)$ converges for all real $x$. First, for $x_{0} \in((j-1) / n, j / n), j \in \mathbf{Z}$, we may argue as for the mid-point collocation method in [4]: Because $d>\beta-1 / 2$, we see from (3.7) and (1.8) that $u_{n} \in H^{\beta}$. Because $u_{h}$ is also smooth in a neighbourhood of $x_{0}$, it then follows, by application of Lemma 3.2 (c) of [4], that $L u_{h}$ is Hölder continuous in a neighbourhood of $x_{0}$. Thus, the Fourier series for $L u_{h}$, which is the right-hand side of (3.8), converges at $x_{0}$ (in the sense of (2.5)) to $L u_{h}\left(x_{0}\right)$. On the other hand, for $x=j / n, j \in \mathbf{Z}$, the right-hand side of (3.8) converges because the sum over $p$ is over the finite set $A_{n}{ }^{*}$ and the sum over $l$ can be written (remembering (2.5)!) as

$$
\begin{aligned}
& \sum_{l \in Z^{*}} \operatorname{sign} l|p+\ln |^{\beta-d-1} \mathrm{e}^{2 \pi i(p+l n) j / n} \\
& =\mathrm{e}^{2 \pi i p j / n} \sum_{i=1}^{\infty}\left[(\ln +p)^{-\alpha}-(l n-p)^{-\alpha}\right] \\
& =\mathrm{e}^{2 \pi i p j / n} n^{-\alpha} \sum_{i=1}^{\infty} l^{-\alpha} \cdot\left[\left(1+\frac{y_{p}}{2 l}\right)^{-\alpha}-\left(1-\frac{y_{p}}{2 l}\right)^{-\alpha}\right],
\end{aligned}
$$

where we have introduced

$$
\alpha=d+1-\beta \geqslant 1 / 2
$$

and

$$
y_{p}=2 p / n \in[-1,1]
$$

Since the mean-value theorem gives

$$
\left(1+\frac{y}{2 l}\right)^{-\alpha}-\left(1-\frac{y}{2 l}\right)^{-\alpha}=-\frac{\alpha}{2 l} y\left[\left(1+\frac{\theta y}{2 l}\right)^{-\alpha-1}+\left(1-\frac{\theta y}{2 l}\right)^{-\alpha-1}\right]
$$

where $0<\theta(y)^{\prime}<1$, it follows that $\left|(1+y / 2 l)^{-\alpha}-(1-y / 2 l)^{-\alpha}\right| \leqq c / l$. Thus the last sum in (3.9) converges, and the convergence of the series for $\bar{L} u_{h}(x)$ has been proved for all $x$.

(It may be remarked that the convergence of the series for $L \mu_{h}$ at the breakpoints occurs in the present work, but not in general in [4], because we have here restricted attention to operators $L$ of even 'symbol' - that is, the' quantity that multiples $\hat{u}(k)$ in (i.3) is an even function: of $k$.)

Since the series (3.8) for $L u_{h}(x)$ converges for all $x$, it follows that the quadrature sum on the left-hand side of (3.1) is well defined for any choice of the quadrature parameters. Now, using (3.2), (3.3), (3.8), (3.10) and (3.11) we obtain, for the lefthand side of (3.1),

$$
Q_{h}\left(\bar{\varphi}_{p} L u_{h}\right)= \begin{cases}\hat{u}_{h}(0), & , p=0,- \\ |p|^{\beta} \hat{u}_{h}(p) D\left(y_{p}\right), . & p \in \Lambda_{n}^{*}\end{cases}
$$

where

and

$$
D(y)=1+\operatorname{sign} y|y|^{\alpha} E(y), \quad . \quad y \in[-1,1]
$$

$$
\begin{aligned}
E(y) & =\sum_{l \in \mathbf{Z}^{*}} \operatorname{sign} l|y+2 l|^{-a} e_{l} \\
& =\sum_{l=1}^{\infty}\left[(2 l+y)^{-a} e_{l}-(2 l-y)^{-a} e_{-l}\right], \quad y \in[-1,1],
\end{aligned}
$$


where $e_{l}$ is given by (3.4). Since the left-hand side of (3.12) is a well defined convergent series, the series $E(y)$ in the definition of $D(y)$ must also converge at least for $y=y_{p}$ $=2 p / n$; i.e. it must converge for all non-zero rational numbers $y$ in $(-1,1]$. The following lemma establishes that in fact (3.14) converges, and therefore $E(y)$ and $D(y)$ are well defined; for all $y \in[-1,1]$. (In this lemma the assumption on $\alpha$ is weakened to $\alpha>0$, since this may be done without cost.)

Lemma 3.1: For $\alpha>0$, consider the series

$$
\sum_{l=1}^{\infty}(2 l+\dot{y})^{-a} c_{l}, \quad y \in[-1,1]
$$

where $\left|c_{l}\right| \leqq m$, with $m$ independent of $l$. If the series converges for some $y_{0} \in[-1,1]$, then it does so for all $y \in[-1,1]$, and the resulting function belongs to $C^{\infty}[-1,1]$.

Proof: The formal derivative of the series (3.15) is (absolutely) uniformily convergent, and can therefore be integrated term by term. Thus for $y \in[-1,1]$ we have

$$
\int_{y_{0}}^{y}\left[-\alpha \sum_{l=1}^{\infty}(2 l+t)^{-\alpha-1} c_{l}\right] d t=\sum_{l=1}^{\infty}\left[(2 l+y)^{-\alpha}-\left(2 l+y_{0}\right)^{-\alpha}\right] c_{l} .
$$

Denoting the series (3.15) by $F(y)$, we know that $\sum_{l=1}^{\infty}\left(2 l+y_{0}\right)^{-a} c_{l}=F\left(y_{0}\right)$ is a convergent sum, from which it now follows that-the, right-hand side of (3.16) can be expressed as the difference of two convergent series. Thus $\sum_{l=1}^{\infty}(2 l+y)^{-a} c_{l}=F(y)$ is
also a convergent sum. Then from (3.16) we have

$$
F^{\prime}(y)=-\alpha \sum_{l=1}^{\infty}(2 l+y)^{-\alpha-1} c_{l}, \quad y \in(-1,1)
$$

remembering that because the latter series is uniformly convergent, it defines a continuous function on $[-1,1]$. Thus we have $F \in C^{\mathbb{1}}[-1,1]$. A similar (but simpler) argument now yields

$$
F^{\prime \prime}(y)=\alpha(\alpha+1) \sum_{l=1}^{\infty}(2 l+y)^{-\alpha-2} c_{l}
$$

and $F \in C^{2}[-1,1]$; and so on. Thus $F \in C^{\infty}[-1,1]$, and the result is proved

Corollary 3.2: With $\alpha>1 / 2, w_{1}, w_{2} \in \mathbf{R}$ and $\varepsilon_{1}, \varepsilon_{2} \in[0,1]$, the series expression (3.14) for $E(y)$ converges for all $y \in[-1,1]$. Moreover, $E \in C^{\infty}[-1,1]$. Thus $D$ is $a$ - continuous function on $[-1,1]$, and is smooth outside an arbitrary neighbourhood of zero.

As in [4] and [14], the properties of the function $D$ are crucial for both the stability and the rate of convergence of the method. To study the stability, it is convenient to write, using (3.4), (3.13) and (3.14);

where

$$
D(y)=w_{1} Z_{\varepsilon_{1}}(y)+w_{2} Z_{\varepsilon_{1}}(y), \quad \cdot \dot{y} \in[-1,1]
$$

$$
Z_{\iota}(y)=1+\operatorname{sign} y|y|^{a} \sum_{l \in Z^{*}} \operatorname{sign} l|y+2 l|^{-a} \mathrm{e}^{2 \pi 1 \varepsilon l}, \quad y \in[-1,1] .
$$

Since $Z_{c}$ is the special case of $D$ obtained by setting $w_{2}=0, w_{1}=1, \varepsilon_{1}=\varepsilon$, it follows from Corollary 3.2 that, for arbitrary $\varepsilon \in[0,1], Z_{\varepsilon}$ is a well-defined and continuous function on $[-1,1]$, and is smooth outside an arbitrary neighbourhood of zero. 
For the stability of the method, the requirement; as in [4] and [14], is that $\dot{D}$ be bounded away from zero. This will follow from the following lemma the rather technical appearance of which is dictated by our desire to obtain explicit bounds for the stability constants).

Lem ma 3.3: Let $\alpha$ and let $Z$, be the complex-valued function defined by (3.17).

(i) For arbitrary $\varepsilon \in(0,1), \operatorname{Re} Z_{\varepsilon}(y) \geqq\left(1-3^{-a}\right)(1-\max (\cos 2 \pi \varepsilon, 0))>0$, $y \in[-1,1]$.

(ii) For $\varepsilon=0, Z_{0}(y)>0, y \in(-1,1), Z_{0}( \pm 1)=0$.

Proof: For arbitrary $\varepsilon \in[0,1]$ we have, from the definition (3.17),

$$
\begin{aligned}
\operatorname{Re} \dot{Z}_{\varepsilon}(y) & =1+\operatorname{sign} y|y|^{\alpha} \sum_{l \in \mathbf{Z}^{*}} \operatorname{sign} l|y+2 l|^{-\alpha} \cos 2 \pi \varepsilon l \\
& =1+\operatorname{sign} y|y|^{\alpha} \sum_{l=1}^{\infty}\left[(2 l+y)^{-a}-(2 l-y)^{-\alpha}\right] \cos 2 \pi \varepsilon l
\end{aligned}
$$

which is manifestly even. For $y \in[0,1]$ we define $h_{l}(y)=y^{a}\left[(2 l+y)^{-\alpha}\right.$ i $\left.(2 l,-y)^{-\alpha}\right]$. Then for $y \in[0,1]$ we have

$$
\operatorname{Re} Z_{\varepsilon} \ddot{(y)}=1+\sum_{l=1}^{\infty} h_{l}(y) \cos 2 \pi \varepsilon l \geqq 1+\sum_{l=2}^{\infty} h_{l}(y)+h_{1}(y) \cos 2 \pi \varepsilon,
$$

because $h_{l}(y) \leqq 0$ and $\cos 2 \pi \varepsilon l \leqq i$. As in (3.9); an application of the mean-value theorem shows that $\sum h_{l}(y)$ is absolutely and uniformly convergent in $[0,1]$. Moreover, because

$$
h_{l}^{\prime}(y)=2 l \alpha y^{\alpha-1}\left[(2 l+y)^{-\alpha-1}-\left(2 l^{\prime}-y\right)^{-\alpha-1}\right]<0, \quad y \in(0,1],
$$

- we see that $h_{l}(y)$ is a decreasing function on $[0,1]$, thus

$$
\begin{aligned}
\operatorname{Re} Z_{c}(y) & \geqq 1+\sum_{l=2}^{\infty} h_{l}(1)+h_{1}(1) \max (\cos 2 \pi \varepsilon, 0) \\
& =1-3^{-a}-\left(1-3^{-\alpha}\right) \max (\cos 2 \pi \varepsilon, 0),
\end{aligned}
$$

and the proof of part (i) of the theorem is complete.

For the case $\varepsilon=0$ we see that $Z_{0}(y)$ is real, thus the conclusion arrived at so far can be expressed as $Z_{0}(y) \geqq 0$. Now since $h_{l}(y)>h_{l}(1)$ for $y \in(0,1]$, it follows from (3.18) and (3.19) that the inequality $Z_{0}(y) \geqq 0$ is strict for $y \in(-1,1)$. Finally,

$$
Z_{0}( \pm 1)=1+\sum_{l=1}^{\infty} h_{l}(1)=0
$$

Remurk : The second purt of the lemma was proved, in effect, in [4, Lemma 2.3] in a different context - see the case of the function $g$ and splines of odd degree.

Corollary 3.4: Let $\alpha>1 / 2$.

(i) With $w_{1}, w_{2} \geqq 0$, and arbitrary $\varepsilon_{1}, \varepsilon_{2} \in[0,1]$, the function $D$ defined by (3.13) and - (3.14) sulisfies.

$$
\begin{aligned}
\operatorname{Re} D(y) \geqq & \left(1-3^{-a}\right)\left[w_{\mathrm{r}}\left(1-\max \left(\cos , 2 \pi \varepsilon_{1}, 0\right)\right)\right. \\
& \left.+w_{2}\left(1-\max \left(\cos 2 \pi \varepsilon_{2}, 0\right)\right)\right], \quad y \in[-1,1] .
\end{aligned}
$$

(ii) If $\varepsilon_{1}=0$ and $w_{1}=1, w_{2}=0$ then $D( \pm 1)=0$. 
Remark: The first part of this result establishes the stability of the method under the conditions stated in the theorem (effectively, that the weights are non-negative and there is at least one quadrature point in the interior). The second' establishes that the latter condition is necessary: in effect it expresses the well known fact that the method of collocation at the break points is unstable in the case of even-degree splines.

Now we turn to the question of the rate of convergence. As in [4] and [14], the maximum rate of convergence is determined by the behaviour of the function $D(y)$ in the neighbourhood of 0 .

Initially we allow the quadrature parameters $\varepsilon_{1}, \varepsilon_{2}$ to be chosen arbitrarily.

Lemma 3.5: With $\alpha>1 / 2, w_{1}, w_{2} \in \mathbf{R}$, and $\varepsilon_{1}, \varepsilon_{2} \in[0,1]$, the function $D$ defined by (3.13) and (3.14) satisfies $|D(y)-1| \leqq C|y|^{a}, y \in[-1,1]$, where $C$ depend's on $\alpha, w_{1}, w_{2}, \varepsilon_{1}, \varepsilon_{2}$, but not on $y$.

Proof: This follows immediately from (3.13) and Corollary 3.2

Next, we consider the quadrature parameters to be chosen symmetrically, as in part (ii) of the theorem: that is, $w_{1}=w_{2}=1 / 2$, and $\varepsilon_{2} \doteq 1-\varepsilon_{1}$. The special feature in this case is that the function $E(y)$ defined by (3.14) now vanishes at $y=0$ : in fact we have, from (3.4), $e_{i}=e_{-l}=\cos 2 \pi \varepsilon_{1} l$, and hence

$$
E(y)=\sum_{l=1}^{\infty}\left[(2 l+y)^{-\alpha},-(2 l-y)^{-\alpha}\right] \cos 2 \pi \varepsilon_{1} l
$$

so that the property $E(0)=0$ is immediately apparent. By the mean-value theorem, we may write

$$
(2 l+y)^{-\alpha}-(2 l-y)^{-\alpha}=-\alpha y\left[(2 l+\theta y)^{-\alpha-1}+(2 l-\theta y)^{-\alpha-1}\right],
$$

where $0<\theta(y)<1$, thius

$$
\begin{aligned}
|E(y)| & \leqq \sum_{l=1}^{\infty}\left|(2 l+y)^{-\alpha}-(2 i-y)^{-\alpha}\right| \\
& \leqq \sum_{l=1}^{\infty}|\alpha||y| 2(2 l-1)^{-\alpha-1}=C|y|
\end{aligned}
$$

Since $D$ is related to $E$ by (3.13), we obtain the following lemma.

Lemma 3.6: With $\alpha>1 / 2, w_{1}=w_{2}=1 / 2, \varepsilon_{2}=1-\varepsilon_{1}$, and $\varepsilon_{1} \in[0,1]$, the function $D$ defined by (3.13). satisfies $|D(y)-1| \leqq C|y|^{\alpha+1}, y \in[-1,1]$, where $C$ depends on $\alpha$ and $\varepsilon_{1}$, but not on $y$.

The function $E(y)$ for the symmetric case can be subjected to a more precise analysis, leading to the parameter choice in part (iii) of the theorem. Replacing the mean-value expression (3.21) by the higher-order version

$$
\begin{aligned}
& (2 l+y)^{-\alpha}-(2 l-y)^{-\alpha} \\
& =-\alpha 2^{-\alpha} l^{-\alpha-1} y-\frac{\alpha(\alpha+1)(\alpha+2)}{6} y^{3}\left[(2 l+0 y)^{-\alpha-3}+(2 l-\theta y)^{-\alpha-3}\right]
\end{aligned}
$$

where $0<\theta(y)<1$, we obtain.from (3.20)

$$
\begin{aligned}
E(y)= & -\alpha 2^{-\alpha} y \sum_{l=1}^{\infty} l^{-\alpha-1} \cos 2 \pi \varepsilon_{1} l \\
& -\frac{\alpha(\alpha+1)(\alpha+2)}{6} y^{3} \sum_{l=1}^{\infty}\left[(2 l+\theta y)^{-\alpha-3}+(2 l-\theta y)^{-\alpha-3}\right] \cos 2 \pi \varepsilon_{1} l
\end{aligned}
$$


If $\varepsilon_{\mathrm{f}}$ is chosen as in part (iii) of Theorem 2.1 then the first term of this expression vanishes, and we have

$$
|E(y)| \leqq \frac{\alpha(\alpha+1)(\alpha+\dot{2})}{6}|y|^{3} \sum_{l=1}^{\infty} 2(2 l-1)^{-\alpha-3}=C|y|^{3} .
$$

Thus we obtain, using (3.13), the following lemma.

Lemma 3.7: With $\alpha>1 / 2, w_{1}=w_{2}=1 / 2,{ }^{\prime}{ }^{\prime}{ }_{2}=1-\varepsilon_{1}$, and $\varepsilon_{1}$ the least positive zero of $G_{\alpha+1}$, defined by (2.3), the function $D$ defined by (3.13) satisfies. $|D(y)-1|$ $\leqq C|y|^{\alpha+3}$, where $C$ depends on $\alpha$, but not on $y$.

In the sequel wie make use of whichever of the three Lemmas 3.5-3.7. is appropriate.

\section{Proof of Theorem 2.1 - final stage}

Now we are ready to establish the existence of $u_{h}$, and prove the estimates (2.1), (2.2) and (2.4) for $\left\|u_{h}-u\right\|_{s}$. Recall that the qualocation method is expressed by (3.1). $\dot{W}$ ith the aid of (3.5) and (3.12), this is expressible as the set of equations.

$$
\begin{aligned}
& \hat{u}_{h}(0)=\hat{u}(0)+\sum_{l \in \mathbf{Z}^{*}}|\ln |^{\beta} \hat{u}(l n) e_{l}, \quad p=0, \\
& |p|^{\beta} \hat{u}_{h}(p) D\left(y_{p}\right)=|p|^{\beta} \dot{\hat{u}}(p)+\sum_{l \in \mathbf{Z}^{*}}|p+\ln |^{\beta} \dot{u}(p+l n) e_{l}, \quad p \in \Lambda_{n}^{*}
\end{aligned}
$$

The subsequent analysis is almost the same as in [14], thus we shall be brief.

Since $D(y) \neq 0$ for $y \in[-1,1]$, the above equations uniquely determine $\hat{u}_{n}(p)$ for all $p \in \Lambda_{n}$. The recurrence relation (3.7) then determines all other Fourier coefficients of $u_{h}$. Thus the approximate solution $u_{h}$ exists and is unique for every continuous right-hand side function $f$. To study the convergence of $u_{h}$ to $u$, we investigate, for $s<d+1 / 2$,

$$
\begin{aligned}
\left\|u_{h}-u\right\| \|_{s}^{2}= & \left|\hat{u}_{h}(0)-\hat{u}(0)\right|^{2}+\sum_{k \in \mathbf{Z}^{*}}|k|^{2 s}\left|\hat{u}_{h}(k)-\hat{u}(k)\right|^{2} \\
\leqq & \left|\hat{u}_{h}(0)-\hat{u}(0)\right|^{2}+2 \sum_{k \notin \Lambda_{n}}|k|^{2 s}|\dot{\hat{u}}(k)|^{2} \\
& +2 \sum_{k \notin \Lambda_{n}}|k|^{2 s}\left|\hat{u}_{h}(k)\right|^{2}+\sum_{p \in A_{n}}|p|^{2 s}\left|\hat{u}_{h}(p)-\hat{u}(p)\right|^{2}
\end{aligned}
$$

For $u \in H^{t}$, and $\beta+1 / 2<t$, it follows as in [14] that the first term of (4.3) is bounded by

$$
\left|\hat{u}_{h}(0)-\hat{u}(0)\right|^{2} \leqq C h^{2(t-\beta)}\|u\|_{t}^{2} .
$$

Similarly, because $s \leqq t$ we obtain as in [14] the bound

$$
\because \quad 2 \sum_{k \in A_{n}}|k|^{28}|\hat{u}(k)|^{2} \leqq C h^{2(t-8)}\|u\|_{t}^{2}
$$

for the second term of (4.3). For the third term of (4.3) it is necessary, as in [14]; to first make use of the recurrence relation (3.7) to express $a_{h}(k)$ in terms of $a_{h}(p)$, with $p \in \Lambda_{n}{ }^{*}$; and then to use (4.2) to express $\hat{u}_{h}(p)$ in terms of Fourier coefficients of $u$. It is in the latter phase that it becomes essential to assume, as in the statement of the theorem, and as we shall assume from now on, that at least one of $\varepsilon_{1}, \varepsilon_{2}$ is in the open interval $(0,1)$, and has associated with it, a positive weight. Then from Corollary 3.4 we have $\left|D(y)^{-1}\right| \leqq C(\alpha)$, and as in [14] it follows that the third term of (4.3) has 
the bound

$$
\begin{aligned}
2 \sum_{k \notin A_{n}}|k|^{2 s}\left|\alpha \hat{u}_{h}(k)\right|^{2} & =2 \sum_{p \in \Lambda_{n}^{*}} \sum_{l \in Z^{*}}|p+\ln |^{2 s}\left|\hat{u}_{h}(p+\ln )\right|^{2} \\
& =2 \sum_{p \in A_{n}^{*}}|p|^{2(d+1)}\left|\hat{u}_{h}(p)\right|^{2} \sum_{l \in Z^{*}}|p+\ln |^{2(s-d-1)} .
\end{aligned}
$$

Because $s<d+1 / 2$, the last sum can be estimated by

$$
\sum_{l \in \mathbf{Z}}|p+\ln |^{2(s-d-1)} \leqq C(s) n^{2(s-d-1)}
$$

see [4]. Next, we use (4.2) and obtain

$$
\begin{aligned}
& 2 \sum_{k \in A_{n}}|k|^{2 s}\left|\hat{u}_{h}(k)\right|^{2} \\
& \leqq C n^{2(s-d-i)} \sum_{p \in A_{n}^{*}}|p|^{2(d+1)} \cdot\left[|\hat{u}(p)|^{2}+|p|^{-2 \beta}\left(\left.\sum_{l \in \mathbf{Z}^{*}}|p+\ln |\right|^{\beta}|\hat{u}(p+\ln )|\right)^{2}\right] \\
& \leqq C n^{2(s-\imath)} \sum_{p \in A_{n}^{*}}|p|^{2 \ell}|\hat{u}(p)|^{2} \\
& \because+C n^{2(s-d-1)} \sum_{p \in \Lambda_{n}^{*}}|p|^{2(d+1-\beta)} \sum_{m \in Z^{*}}|p+\dot{m} n|^{2(\beta-t)} \sum_{t \in \mathbf{Z}^{*}}|p+\ln |^{2 \ell}|\hat{u}(p+\ln )|^{2},
\end{aligned}
$$

where $\tau=\min (d+1, \iota)$.

Hence, because $\beta+1 / 2<\ell$,

$$
\begin{aligned}
& 2 \sum_{k \in \Lambda_{n}}|k|^{2 s}\left|\hat{u}_{h}(k)\right|^{2} \\
& \leqq C n^{2(s-n)}\|u\|_{t^{2}}+C n^{2(s-d-1+\beta-\theta)} \sum_{\sum_{p} \in \Lambda_{n}^{*}}|p|^{2(d+1-\beta)} \sum_{t \in \mathbf{Z}^{*}}|p+\ln |^{2 t}|\hat{u}(p+\ln )|^{2} \\
& \leqq\left(C n^{2(s-t)}+C^{\prime} n^{2 !(s-t)}\right)\|u\|_{t}^{2} \text {, }
\end{aligned}
$$

or

$$
2 \sum_{k \notin A_{n}}|k|^{2 s}\left|\hat{u}_{h}(k)\right|^{2} \leqq C h^{2(r-s)}\|u\|_{t}^{2}
$$

Finally, we consider the fourth term of (4.3). This is the term that plays the crucial rôle.in limiting the maximum attainable rate of convergence. Using (4.2) we have

$$
\hat{u}_{h}(p)-\hat{u}(p)=D\left(y_{p}\right)^{-1}\left[\left(1-D\left(y_{p}\right)\right) \hat{u}(p)+|p|^{-\beta} \sum_{i \in \mathbf{Z}^{*}}|p+\ln |^{\beta} \hat{u}(p+\ln ) e_{i}\right] .
$$

Then with the aid of the stability property in Corollary 3.4, and also whichever of the three Lemmas $3.5-3.7$ is appropriate, together with $y_{p}=2 p / n$, we obtain

$$
\begin{aligned}
\left|\hat{u}_{h}(p)-\hat{u}(p)\right|^{2} \leqq & C\left(\frac{p}{n}\right)^{2(d+1-\beta+r)}|\hat{u}(p)|^{2} \\
& +C|p|^{-2 \beta} \cdot\left(\sum_{l \in \mathbf{Z} \cdot}|p+\ln |^{\beta}|\hat{u}(p+\ln )|\right)^{2}
\end{aligned}
$$

where $r=0,1$ or 3 , depending on whether the quadrature parameters are as in part . (i), (ii) or (iii) of the theorem. It then follows, by the same argument as in [14], that the fourth term of (4.3) satisfies

$$
\sum_{p \in A_{n^{*}}}|p|^{2 \delta}\left|\hat{u}_{h}(p)-\hat{u}(p)\right|^{2} \leqq C h^{2 \min (t-s, t-\beta . d+1+r-\beta)}\|u\|_{t^{2}} .
$$


Collecting together the -separate bounds $(4.4),(4.5) ;(4.6)$ and $(4: 7)$,. we obtain

$$
\left\|u_{b}-u\right\|_{s}^{\prime} \leqq C h^{\min (t-s . t-\beta . d+1-s, d+1+r-\beta)}\|u\|_{t},
$$

and the proof. of Theorem 2.1 is completed by inserting in this bound the appropriate values of $r$

\section{The function $G_{y}$}

Since the function $G_{y}$, defined by

$$
G_{r}(x)^{\prime}=\sum_{n=1}^{\infty} n^{-\gamma} \cos 2 \pi n x^{\prime}, \quad x \in \mathbf{R}, \gamma>1,
$$

plays a key rôle in the theory, we note briefly some of its properties.

Since $\gamma>1$, the series (5.1) is (absolutely) uniformly convergent, and therefore $G$, is a continuous, even, 1-periodic function on $\mathbf{R}$. Moreover,

$$
\begin{aligned}
& G_{\gamma}(0)=\sum_{n=1}^{\infty} \frac{1}{n^{\gamma}}=\zeta(\gamma)>0, \\
& G_{\gamma}\left(\frac{1}{2}\right)=\sum_{n=1}^{\infty} \frac{(-1)^{n}}{n^{\gamma^{\prime}}}=-\left(1-2^{1-\gamma}\right) \zeta(\gamma)<0,
\end{aligned}
$$

where $\zeta$ is the Riemann zeta function, and where the last step follows, for example, from $J_{A H N K}$ and Emwe [8, p. 319]. Since the continuous function $G_{\gamma}$ changes sign on $(0,1 / 2)$, it has at leást one zero in that interval.

If $\gamma$ is an even integer, then $G_{\nu}$ is closely related to a Bernoulli polynomial: in fact for $\gamma=2,4, \ldots$ we have $[1$, p. 805]

$$
G_{\gamma}(x)=(-1)^{1+\gamma / 2} \frac{2^{y-1} \pi^{\gamma}}{\gamma !} B_{\gamma}(x), \quad x \in[0,1] .
$$

Here the uniqueness of the zero on $(0,1 / 2)$ is apparent from the known behaviour of Bernoulli polynomials: The following result establishes that there' is a unique zero on $(0,1 / 2)$ for all real $\gamma \geqq 1$.

Theo re m 5.1 [5]: For $\gamma \geqq 1$ the function $G_{\gamma}$ defined by $(5.1)$ is decreasing on $(0,1 / 2)$, and has a unique zero $x_{0}(\gamma) \in(0,1 / 2)$, which satisfies

$$
\lim _{\gamma \rightarrow \infty} x_{0}(\gamma)=\dot{1} / 4 \text {. }
$$

\section{(j. Qualocation in the presence of a smoother perturbation}

In this section we apply the same qualocation method to the more general pseudodifferential equation which can be written as perturbed equation

$$
(L+K) u=\ell,
$$

where $L$ is as in (1.3), and $K$ is an operator having a greater smoothing effect than $L$.

The following result is stated initially in a more gerieral form than we need for our present púrposes. The proof is modelled on 'one used by ARNoL.D' and WENDr.AND [4] for the case of the collocation.method. 
Theorem 6.1: Let $\beta$ be a real number, and assume that $L$ is an isomorphism from $H^{s}$ onto $H^{s-\beta} \forall s \in\left[\beta-a, \mu+d+\frac{1}{2}\right)$, with $\mu, d$ and a as given below. Let $\mu>0$ be such that $K$ maps $H^{s-\mu}$ boundedly into $H^{s-\beta}$ for the above range of s values, and assume $L+K$ is bijective from $H^{s}$ onto $H^{s-\beta}$. Assume also that a given qualocation method, with trial space. $S_{h}$ consisting of smoothest splines of degree $d>\beta-\mu$, and with given test space $T_{h}$ and quadrature rule $Q_{h}^{\prime}$, has the following property when applied to the equation

$$
L w \stackrel{:=}{=} ; \quad f \in H^{t-\beta}, \therefore w \in H^{t} .
$$

namely that the qualocation approximation $w_{h} \in S_{h}$ exists uniquely for $h \leqq h_{0}$, with $h_{0}$ , independent of $f$, and satisfies, for some fiẍed $a \geqq 0$,

$$
\left\|w_{h}-w\right\|_{s} \leqq C h^{\min (t-s, t-\beta, d+1-8, d+1+a-\beta) \cdot}\|w\|_{t} .
$$

for all real.s, $t$ satisfying

$$
\beta-a \leqq s \leqq t, \quad s<d+1 / 2, \quad \beta+1 / 2<t .
$$

Then the same result holds, provided $t$ satisfies also

$$
\mu+\beta-a \leqq t<\mu+d+1 / 2,
$$

for the qualocution method $\left\{S_{h}, T_{h}, Q_{h}\right\}$ applied to the equation

$$
(L+K) u=f, \quad \dot{f} \in H^{i-\beta}, \quad u \in H^{t} .
$$

Stated fully, the result is that the qualocation approximation $u_{h_{j}} \in S_{h}$ exists uniquely for $h$, sufficiently small, and satisfies

$$
\left\|u_{h}-u\right\|_{s} \leqq C h^{\min (t-s, t-\dot{\beta}, d+1-s, d+1+a-\beta)}\|u\|_{t}
$$

for all s, 't salisfying (6.4) and (6.5).

Proof: Take $h \leqq h_{0}$, and assume provisionally that $u_{h}$, a qualocation solution to (6.6), exists. Then by definition $u_{h} \in S_{h}$ satisfies, for arbitrary $\dot{\chi}_{h} \in T_{h}^{\prime}$ '.

or

$$
Q_{h}\left(\bar{\chi}_{h}(\dot{L}+K)\left(u-u_{h}\right)\right) \stackrel{:}{=} 0,
$$

where

$$
Q_{h}\left(\bar{\gamma}_{h} L u_{h}\right)=Q_{h}\left(\bar{\chi}_{h}\left[L u+K\left(u-u_{h}\right)\right]\right)=Q_{h}\left(\bar{\chi}_{h} L w\right)
$$

$$
w=u+L^{-1} K\left(u-u_{h}\right) .
$$

It follows from the assumption, since $\chi_{h} \in T_{h}$ is arbitrary, that $u_{h}$ is the unique qualocation approximation to an equation of the form (6.2) for which the exact solution is (6.8). Then the estimate (6.3) gives

$$
\begin{aligned}
& \left\|u-u_{h}+L^{-1} K\left(u-\dot{u_{h}}\right)\right\|_{s} \\
& \leqq C h^{\min (t-s, t-\beta, d+1-s, d+1+a-\beta}\left\|u+L^{-1} K\left(u-u_{h}\right)\right\|_{t},
\end{aligned}
$$

. for $s, i$ satisfying (6.4). Assume that $t$ satisfies also (6.5). Then it follows from the mapping properties of $L$ and $K$ that

$$
\begin{aligned}
\left\|\bar{u}+L^{-1} K\left(u-u_{h}\right)\right\|_{t} & \leqq\|u\|_{t}+C\left\|K\left(u-u_{h}\right)\right\|_{t-\beta} \\
& \leqq\|u\|_{t}+C\left\|u-u_{h}\right\|_{t-p} .
\end{aligned}
$$


We now obtain, using (6.9), (6.10) and the assumed mapping property of $K+L$,

$$
\begin{aligned}
\left\|u-u_{h}\right\|_{s} & \leqq C\left\|(L+K)\left(u-u_{h}\right)\right\|_{s-\beta} \\
\therefore \quad & \leqq C\left\|L^{-1}(L+K)\left(u-u_{h}\right)\right\|_{s}=C\left\|\left(l+L^{-1} K\right)\left(u-u_{h}\right)\right\|_{s} \\
& \leqq C h^{\min (t-s, d-\beta, d+1-8, d+1+a-\beta)}\left(\|u\|_{l}+\left\|u-u_{h}\right\|_{l-\mu}\right) .
\end{aligned}
$$

In particular, setting $s=t-\mu$ we obtain

$$
\left\|u-u_{h}\right\|_{t-\mu} \leqq C h^{\dot{m}\{\operatorname{m}(\mu, t-\beta, \dot{\mu}+d+1-t, d+1+a-\beta)}\left(\|u\|_{t} \dot{+}\left\|u-\dot{u}_{h}\right\|_{t-\mu}\right) .
$$

Since the exponent of $h$ is positive because of the assumptions (6.4) and (6.5), for $h$ sufficiently small (say $h \leqq h_{1}$ ) we have

$$
\left\|u-u_{h}\right\|_{t-\mu} \leqq C h^{\min (\mu, \ell-\beta, \mu+d+1-t, d+1+a-\beta}\|\dot{u}\|_{t} .
$$

Thus the second term of (6.11) is of higher order than the first, and so (6.11) yields the desired result (6.7).

To complete the proof, we now observe from (6.7) that the qualocation solution $u_{h}$ is necessarily unique for $h \leqq h_{1}$. Thus the corresponding linear equations are of full rank, from which it follows that the qualocation solution exists for every continuous function $f$.

It follows from the theorem that if $\mu$ (which is related to the smoothing property of $K$ ) is smaller'than $a+1 / 2$, then the maximum rate of convergence of $\left\|u_{h}-u\right\|_{s}$; for any value of $s$, is reduced from $O(h)^{(d+1+\alpha-\beta)}$ to $O\left(h^{d+1 / 2+\mu-\varepsilon-\beta)}\right)$, where $\varepsilon>0$ is arbitrary. On the other hand, if $K$ is a perturbation arising from the mapping of the logarithmic potential from a $C^{\infty}$, curve to the unit circle, then $\mu$ can typically be taken arbitrarily large, and then no reduction in the maximum order will occur.

We now specialize to the case of even-degree splines, and the particular qualocation method of this paper.

Coroliary 6.2: Let $\beta, L, d, S_{h}, T_{h}$ and $Q_{h}$ be as in Theorem 2.1. Let $t>\beta+1 / 2$ and $\mu>0$ be such that $K$ maps $H^{s-\mu}$ boundedly into $H^{s-\beta}$ for $s \dot{\epsilon}[\beta-a, \mu+d+1 / 2)$, and assume that $L+K$ is bijective from $H^{s}$ onto $H^{s-\beta}$ for these values of s. A ssume also $f \in H^{t-\beta}$, where $\mu+\beta-a \leqq t<\mu+d+1 / 2$. Let $u \in H^{t}$ be the unique solution of (6.1).

Then for $h$ sufficiently small, the qualocation method $\left\{S_{h}, T_{h}, Q_{h}\right\}$ applied to (6.1) yields a unique approximation $u_{h} \in S_{h}$, and if $s$ and $t$ satisfy (6.4) (with a taking the values $0,1,3$ respectively), then the error norms $\left\|u_{k}-u\right\|_{s}$. satisfy the statements in parts (i), (ii) and (iii) of Theorem 2.1 .

Before concluding, we note that Theorem 6.1 can be applied with equal effect to the case of the odd-degree spline qualocation method considered in [14]. Thus a result analogous to Corollary 6.2 holds also in that case.

\section{REFERENCES}

[1] Abramowitz, M., and L. A. Stequn (eds.): Handbook of Mathematical Functions Washington:'U. S. Department of Commerce 1964.

[2] Arsold, D: N.: A spline-trigonometric Galerkin method and an exponentially convergent boundary integral method. Math. Comp. 41 (1983), 383-397. 
[3] ARnold, D. N., and W. L. Wexdland: Collocation versus Galerkin procedures for boundary integral equations. In: Boundary Element Methods in Engíneering (od,: C. A. Brebbia). Berlin': Springer-Verlag 1982, 18-33.

[4] Arnold, D. N., and W. L. Wendlan: The convergence of spline collocation for strongly elliptic equations on curves. Numer. Math. 47 (1985), 317-341.

[5] Brows, G., and D. WiLsos: Trigonometric sums and polynomial zeros. In : Proceedings of the Centre for Mathematical Analysis. Canberra: Australian National University. To appear.

[6] Hsiao, G. C., Kopp, P., and W. L. WENDIAND: Some applications of a Galerkin-collo'cation method for boundary integral equations of the first kind. Math. Meth. Appl.' Sci: 6 (1984); $280-325$.

[7] Hsiao, G. C., and W. L. Wenduand The Aubin-Ṇitsche lemma for integral equations. J. Int. Equ. 3 (1981), $399-415$.

[8] Jafnke, E., and F. EMde: Tables of Higher Functions. Teubner 1933.

[9] Rathsfelo, A.: Quadraturformelverfahren für ẹndimẹnsionale singuläre Intégralgleichungen. In: Sèminar Analysis, Operator Equations and Numerical Analysiś 1985/86. Berlin: Karl-Weierstra B-Institut für Mathematik (1986), 147-186.

[10] Saranen, J.: The convergence of even degree spline collocation solution for potential problems in smooth domains of the, plane. Numer. Math. 53 (1988), 499-512.

[11] Saranex, J., and W. L. Wendlasd: On the asymptotic convergence of collocation methods with spline functions of even degree. Math. Comp. 45 (1985), $91-108$.

[12] Saranen, J., and W. L. Wenduand: The Fourier series representation of pseudo-differential operators on closed curves. Complex Variables, Theory and Appl. S (1987), 55-64.

[13] Schmint, G.: On spline collocation methods for boundary integral equations in the plane. Math. Meth: Appl. Sci. 7 (1985), 74-89:

[14] Sloan, I. H.: A quadrature-based approach to improving the collocation method. Numer: Math. $54(1988) \div 41-56$.

Manuskripteingang: 11.08. 1988

\section{VERFASSER:}

Prof. Dr. Ian H. Sloax

School of Mathematics, University of New South Wales

Kensingtion NSW 2033, Australia'

Prof. Dr.-Ing. Wolfoasg L. Wendeavi)

Mathematisches Institut A der Universität Stuttgart

Pfaffenwaldring 57

D. 7000 Stuttgart $80^{\circ}$ 